\title{
AÇÃO DA LASERTERAPIA DE BAIXA INTENSIDADE SOBRE PARÂMETROS BIOQUÍMICOS. UMA REVISÃO DE LITERATURA.
}

Heliard Rodrigues dos Santos Caetano ${ }^{1,2}$, Éverton Alex Carvalho Zanuto ${ }^{2,3,4}$.

1- Discente do Curso de Fisioterapia. Universidade do Oeste Paulista (UNOESTE), Presidente Prudente - SP. 2Programa de Pós-Graduação Lato Sensu em Fisiologia, Metabolismo do Exercício e Treinamento. Universidade do Oeste Paulista (UNOESTE), Presidente Prudente - SP. 3- Docente do Curso de Educação Física. Universidade do Oeste Paulista (UNOESTE), Presidente Prudente - SP. 4- Grupo de Investigação Científica Relacionado à Atividade Física (GICRAF). Laboratório de Investigação em Exercício (LIVE); UNESP, Presidente Prudente.

\section{RESUMO}

Terapia Laser de Baixa Intensidade (LLLT) é um recurso fisioterápico amplamente utilizado para cicatrização tecidual, pesquisas recentes buscam novas técnicas de aplicações com objetivos diversificados. Esta pesquisa tem por objetivo realizar um levantamento bibliográfico sobre a LLLT, seus benefícios, mecanismos de ação, modelos de aplicação e comprimentos de ondas; principalmente sua interação nas células musculares, tanto na potencialização do treinamento aeróbio quanto na modulação do nível sérico de enzimas sarcoplasmáticas pós-esforços intensos. Para a realização deste trabalho foram pesquisados periódicos, livros, sites e bases de dados como Bireme, Medline, Scielo e Lilacs com limite de quinze anos, porém alguns bons artigos foram citados com tempos maiores de publicação. Trata-se de uma área nova que ainda não possui parâmetros ideais de aplicação, mas pesquisas recentes vêem aprimorando esta técnica e alcançado resultados cada vez mais satisfatórios como: diminuição de fadiga, controle de dor, redução da lesão muscular oriunda de exercícios físicos, remoção de lactato, modulação dos níveis séricos de CKMM, LDH, e EROs, melhora na ação mitocondrial e aumento na micro circulação. Foi possível concluir que é necessário um maior número de pesquisas voltadas a explicar os mecanismos de interação da foto estimulação com as células musculares de forma mais aprofundada, para que se possa difundir esta técnica que ainda é pouco utilizada.

Palavras-chave: LLLT, Enzimas sarcoplasmáticas, recuperação muscular e Lesão muscular.

\section{INTRODUÇÃO:}

Townes e Schawlow em 1958 propuseram o primeiro modelo para o desenvolvimento de um laser, apoiados no fenômeno físico da emissão estimulada postulado por Albert Einstein em $1916^{2}$, porém o primeiro feixe de luz laser de material sólido, utilizando cristal de rubi com comprimento de onda na faixa vermelha do espectro eletromagnético foi produzido dois anos depois em 1960 por Theodore Maiman, e nas décadas seguintes surgiram vários aparelhos com comprimentos de ondas diferentes, mas baseados no protótipo inicial de Maiman ${ }^{3}$.

O Laser foi desenvolvido inicialmente com alta potência e ficou conhecido como high level laser treatment (HLLT), seus efeitos destrutivos sobre os tecidos foram utilizados para cortar, coagular e cauterizar, somente após muitos anos voltaram-se para aplicações atérmicas, com baixas potências ficando então conhecido como Low Level Laser Therapy (LLLT) ${ }^{4}$. 
Ao nos referirmos à terapia laser de baixa intensidade deve ter em mente uma condição segura e eficaz de tratamento, que se caracteriza como recurso luminoso com propriedades particulares que provocam reações biológicas nos tecidos, gerando ótimos resultados como redução do processo inflamatório, da dor e cicatrização dos tecidos ${ }^{5}$.

O raio laser vermelho é uma modalidade de LLLT que possui maior dificuldade em penetrar a pele, sendo assim mais utilizado em lesões mais superficiais, tornando difíceis pesquisas que objetivam atingir tecidos mais profundos, neste caso o infravermelho demonstra ser mais eficaz ${ }^{6}$.

A LLLT é amplamente conhecida na cicatrização de feridas infectadas, queimaduras, enxerto de pele e amputações por induzir o aumento da atividade mitótica, do número de fibroblastos, da síntese de colágeno e promover a neovascularização ${ }^{7}$.

Em trabalhos recentes com animais notou-se que o laser infravermelho pode ser efetivo no controle de espécies reativas de oxigênio (EROS), através da contenção da isquemia muscular, e no aumento de antioxidantes ${ }^{8,9}$. Outro estudo realizado por Leal Junior et al ${ }^{10}$ notificou que a LLLT aplicada antes do esforço foi capaz de reduzir os danos musculares e acelerar a remoção de lactato.

Apesar de ser uma área nova, diversos pesquisadores estão voltando-se para a LLLT e seus efeitos, em diversas áreas como fadiga, controle de dor, redução da lesão muscular oriunda de exercícios físicos, remoção de lactato, modulação dos níveis séricos de CKMM, LDH, e EROs, melhora na ação mitocondrial e aumento na microcirculação, porém, os parâmetros de aplicação ainda não estão totalmente compreendidos ${ }^{10}$.

Este mesmo autor ${ }^{10}$ cita ainda pesquisadores que encontraram resultados distintos com a LLLT como se segue ${ }^{11}$ : efeitos positivos na fibromialgia e dores musculares no pescoço ${ }^{12}$; na atenuação da fadiga músculo esquelética, e redução da lesão induzida por micro-contrações seqüenciais por estimulação elétrica e $\mathrm{Xu}$ et $\mathrm{al}^{13}$ observaram que a LLLT melhorou a função mitocondrial em células musculares.

Finalizando a LLLT pode também ser utilizada como auxiliar no desempenho aeróbico em atletas, visto que alguns de seus efeitos são semelhantes às adaptações decorrentes de treinamento de longa duração. Podendo ainda ser utilizado no processo de recuperação muscular ${ }^{15}$.

Este estudo tem por objetivo realizar um levantamento bibliográfico sobre a LLLT, seus benefícios, mecanismos de ação, modelos de aplicação e comprimentos de ondas; 
principalmente sua interação nas células musculares, tanto na potencialização do treinamento aeróbio quanto na modulação do nível sérico de enzimas sarcoplasmáticas pós-esforços intensos.

\section{MÉTODOS}

Para a realização deste trabalho foram pesquisados periódicos, livros, sites e bases de dados como Bireme, Medline, Scielo e Lilacs com limite de quinze anos de publicação, porém alguns bons artigos foram citados com dadas anteriores. As palavras chaves utilizadas foram LLLT, Enzimas sarcoplasmáticas, recuperação muscular e Lesão muscular, nos idiomas: português (Brasil), Inglês (Britânico) e Espanhol (Tradicional).

\section{DISCUSSÃO}

Atualmente, tais modelos de recuperação têm sido usados com freqüência, mas seus resultados são questionáveis devido à escassez de informações sobre os aspectos fisiológicos envolvidos no fenômeno e à falta de padronização das rotinas para coleta de informações ${ }^{16}$.

A LLLT é enormemente vantajosa em referência à sua ação menos invasiva, atérmica, indolor, mais barata e com tempo de aplicabilidade inferior aos demais recursos fisioterápicos ${ }^{17}$, sua energia usadas e comprimentos de onda capazes de penetrar nos tecidos ${ }^{29}$.

Resultados encontrados por Martinasso et al $^{18}$ em estudo em vitro onde foi observado o efeito da LLLT na formação óssea em células derivadas de osteoblastos humanos, após segundo dia de repetidas estimulações ocorreu um incremento na multiplicação de células formadoras de nódulos ósseos, osteoblastos e proteínas essenciais para a formação óssea, com um aumento na diferenciação celular que resulta em melhor qualidade do osso formado.

Vários estudos elucidam a interação de moléculas fotorreceptoras (flavoproteinas, porfirinas, citocromo, tirosina e asparaginina, dentre outras) com a LLLT em uma faixa de aplicação próxima a 808nm de comprimento de onda ${ }^{10 ; 13 ; 19,20}$.

Esta alteração no metabolismo celular gerado pelos fotorreceptores absorvendo luz e a transformando em efeitos fotoquímicos, fotofísicos e ou fotobiológicos benéficos possui aplicabilidade infindável. Os antioxidantes ativados por esta técnica podem auxiliar no treinamento esportivo, visto que 2 a 5\% do consumo total de oxigênio geram espécies reativas de oxigênio (EROS) ${ }^{21}$ e que o consumo de oxigênio durante atividades esportivas de longa duração é altíssimo. 
No futebol, por exemplo, $88 \%$ da energia despendida durante uma partida é fornecida pelo metabolismo aeróbio $^{22 ;}{ }^{23}$, considerando estes dados e sabendo que EROS em grande quantidade aumenta o nível de peroxidação dos lipídeos constituintes da membrana celular $^{24}$, e que a destruição da membrana celular acarreta em alteração ou perda de função desta célula, esta técnica pode ser utilizada como profilaxia a lesões por estresse oxidativo em futebolistas.

A aplicação da fototerapia foi capaz de reduzir o dano muscular, através do menor incremento dos níveis séricos de LDH e CK, também encontraram menor atividade da CK após contrações estimuladas eletricamente em músculos de ratos ${ }^{32}$.

A incidência do Laser sobre o tecido inflamado gera vários efeitos benéficos, como aumento da microcirculacao local por vasodilatação (Óxido Nítrico), aumento da permeabilidade vascular e melhora da drenagem linfática, promovendo reabsorção do edema e de substratos catabólitos ${ }^{25}$, assim como controle do efeito cascada da inflamação (liberação de substancias: Prostaglandina E2, prostaciclinas, histamina, serotonina, bradicinina e leucotrienos), gerando uma resposta mais controlada e alivio da dor ${ }^{26 ; 27}$.

$\mathrm{O}$ estudo realizado por $\mathrm{Amat}^{20}$ traz outro aspecto importante, o desempenho aeróbio visto que moléculas fotorreceptoras como o citocromo são importantes na cadeia transportadora de elétrons, assim como as enzimas participantes do sistema oxidativo que têm sua atividade aumentada (modificações químicas). O mesmo autor relata ainda a junção e aumento de mitocôndrias vizinhas (modificações físicas) alterando positivamente a bioenergética com o aumento da síntese de ATP 20 .

Esta priorização do sistema oxidativo foi notificado por Vieira ${ }^{28}$ em estudo sobre o efeito da laserterapia associado ao exercício, onde observou um aumento da atividade da Citrato Sintase, marcador padrão da capacidade aeróbia e uma diminuição da Lactato Desidrogenase, responsável pela reação reversível entre piruvato e lactato.

Possuindo um controle da produção de EROS, uma modulação do processo inflamatório e uma potencialização da síntese de ATP com menor utilização de glicogênio, esta técnica terapêutica consegue reduzir a fadiga ${ }^{19}$, sendo que utilizando os vários tipos de laser, ALGAInP, AsGa, AsGaAL e HeNe ${ }^{30}$, dando condições para evolução do treinamento, assim como a possibilidade de recuperação precoce após esforços intensos. 
Concluindo que os efeitos da laserterapia na fadiga, controle de dor, remoção de lactato, modulação dos níveis séricos de CKMM, LDH, e EROs, melhora na cicatrização, melhora na ação mitocondrial. Podendo agregar uma ação preventiva a fadiga muscular.

\section{CONCLUSÃO}

De acordo com esta revisão de literatura a laserterapia é um grande recurso para diversas áreas, podendo ser utilizado tanto na recuperação quanto no auxilio do condicionamento físico, que ainda não tem parâmetros ideais de aplicação, mas, após estudos bem elaborados em humanos pode futuramente ser introduzido no âmbito esportivo, facilitando o trabalho dos fisioterapeutas e fisiologistas.

\section{REFERÊNCIAS}

1. Kitchen S.S.; Bazin S. Eletroterapia de Clayton. São Paulo: Manole, 191-210, 1996. In VIEIRA, W.H.B. Efeitos do laser de baixa intensidade $(800 \mathrm{~nm})$ sobre a performance muscular de mulheres jovens sob treinamento físico. Tese de Doutorado. Universidade Federal de São Carlos. São Calos: UFISCAR, 2008

2.Tuner J; Hode L. Low Level Laser Therapy. Clinical practice and scientific backgrown. Sweden: Prima Books, 1999.

3. Baxter GD. Therapeutic Lasers: theory and practice. United States of America: Ed. Churchill Livingstone, 1997.

4. Karu T. Primary and secondary mechanisms of action of visible to near-IR radiatiion on cells. J Photochem Photobiol B: Biol, 49:1-17, 1999. http://dx.doi.org/10.1016/S1011-1344(98)00219-X

5. Enwemeka, C.S.; Parker J.C.; Downdy, D.S.; Harkness, E.E.; Sanford, L.E.; Woodrhuff, L.D. The efficacy of Low-Power Lasers in Tissue Repair and Pain Control: A Meta-Analysis Study. Photomed Laser Surg, 22:323-329, 2004. http://dx.doi.org/10.1089/pho.2004.22.323

6. Enwemeka, C. S. Attenuation and penetraition depth of red $632.8 \mathrm{~nm}$ and invisible infrared 904nm light in soft tessui. J Laser Ther, 13:95-101, 2001. http://dx.doi.org/10.5978/islsm.13.95

7. Phaneuef, S.; Leewenburgh, C. Apoptosis and Exercise. Med Sci Sports Exerc: 33:393-396, 2001. http://dx.doi.org/10.1097/00005768-200103000-00010

8. Avni, D.; Levkovits, S.; Maltz, L.; Oron U. Protection of skeletal muscle from ischemic injury: lowlevel laser therapy increases antioxidant activity. Photomed Laser Surg 23:273-277, 2005. http://dx.doi.org/10.1089/pho.2005.23.273 
9. Rizzi C. F.; Mauriz, J. L.; Freitas Correa, D. S.; Moreira, A. J.; Zettler, C. G.; Filippin, L. I. et al. Effects of low-level laser terapy (LLLT) on the nuclear factor (NF) - KappaB signaling pathway in traumatized muscle. Lasers Surg Med. 38:704-713, 2006. http://dx.doi.org/10.1002/lsm.20371

10. Leal Junior, E. C. P. et al. Effect of $830 \mathrm{~nm}$ low-level laser therapy applied before high-intensity exercises on skeletal muscle recovery in athletes. Lasers Med Sci 24:857-863, 2009. http://dx.doi.org/10.1007/s10103-008-0633-4

11. CHOW R.T., HELLER G.Z., BARNSLEY L. The effect of $300 \mathrm{~mW}, 830 \mathrm{~nm}$ laser on chronic neck pain: a double-blind, randomized, placebo-controlled study. Pain 124:201-210, 2006. In LEAL JUNIOR, E. C. P. et al. Effect of $830 \mathrm{~nm}$ low-level laser therapy applied before high-intensity exercises on skeletal muscle recovery in athletes. Lasers Med Sci 24:857-863, 2009.

12. LOPES-MARTINS RA, MARCOS RL, LEONARDO PS et al. Effect of low-level laser (Ga-Al-As 655 $\mathrm{nm}$ ) on skeletal muscle fatigue induced by electrical stimulation in rats. J Appl Physiol 101:283288. In LEAL JUNIOR, E. C. P. et al. Effect of $830 \mathrm{~nm}$ low-level laser therapy applied before highintensity exercises on skeletal muscle recovery in athletes. Lasers Med Sci (2009) 24:857-863, 2009

13. XU X.; ZHAO X.; LIU T.C.; PAN H. Low-intensity laser irradiation improves the mitochondrial dysfunction of $\mathrm{C} 2 \mathrm{C} 12$ induced by electrical stimulation. Photomed Laser Surg 26:197-202, 2008. In LEAL JUNIOR, E. C. P. et al. Effect of $830 \mathrm{~nm}$ low-level laser therapy applied before high-intensity exercises on skeletal muscle recovery in athletes. Lasers Med Sci (2009) 24:857-863, 2009.

14. Vieira, W. H. B. et al. Adaptação enzimática de LDH em ratos submetidos a treinamento aeróbico em esteira e laser de baixa intensidade. Rev Bras de Fisioterapia. 1413-3555, 2006.

15. SUSSAI, D. A.; CARVALHO, P. T. C.; DOURADO, D. M.; BELCHIOR, A. C. G.; REIS, F. A.; PEREIRA, D. M. Low-level laser terapy attenuates creatine kinase levels and apoptosis during forced swimming in rats. Laser Med Sci. doi 10.1007/s10103-009-0697-9, 2009. http://dx.doi.org/10.1007/s10103-009-0697-9

16. PASTRE, M. C.; BASTOS, F. N.; NETTO-JUNIOR, J.; VANDERLEI, L. C. M.; HOSHI, R. A. Métodos de recuperação pós exercícios: uma revisão sitemática. Rev Bras Med Esporte. 1517-8692, 2009.

17. Vinck, E. M. et al. Increased fibroblast proliferation induced by light emitting diode and low power laser irradiation. Lasers in Medical Science, London, v.18, n.2, p.95-99, may 2003. http://dx.doi.org/10.1007/s10103-003-0262-x

18. Martinasso G, Mozzati M, Pol R, Canuto R A, Musio G. Effect of superpulsed laser irradiation on bone formation in a human osteoblast-like cell line. Minerva Stomatol, 56(1-2): 27-30, 2007.

19. Vieira, W. H. B. Efeitos do laser de baixa intensidade $(800 \mathrm{~nm})$ sobre a performance muscular de mulheres jovens sob treinamento físico. Tese de Doutorado. Universidade Federal de São Carlos. São Calos: UFISCAR, 2008. 
20. Amat, A. et. al. Modification of the intrinsic fluorescence and the biochemical behavior of ATP after irradiation with visible and near-infrared laser light. Journal of Photochemistry and Photobiology B: Biology, Lausanne, v. 81, n.1, p. 26-32, oct. 2005.

21. JENKINS, R. R.; GOLDFA. R. B. A. Introduction: oxidant stress, aging and exercise. Medicine \& Science in Sports \& Exercise, Madison, v.25, p.210-12, 1993

22. Shepard RJ. Meeting carbohydrate and fluids needs in soccer. Can J Sports Sci 1990;15:165-71. In Zanuto, E A C. Análise epidemiológica de lesões e perfil físico de atletas do futebol amador na região do oeste paulista. Rev Bras Med Esporte. 2010, vol.16, n.2, pp. 116-120. ISSN 1517-8692

23. EKBLOM, B. Applied physiology of soccer. Sports Med; 3:50-60, 1993. In Zanuto, E A C. Análise epidemiológica de lesões e perfil físico de atletas do futebol amador na região do oeste paulista. Rev Bras Med Esporte. 2010, vol.16, n.2, pp. 116-120. ISSN 1517-8692.

24 ALESSIO, H. M.; GOLDFARB, A. H.; CUTLER, R. G. MDA content increases in fast and slow twitch skeletal muscle with intensity of exercise in a rat. American Journal of Physiology, Washington, v.255, p.C874-77, 1988

25. ALMEIDA-LOPES, L.; LOPES, A. Técnica da drenagem linfatica ativada por laserterapia. In: DIB, L. L.; SADDY, M. S. (Org.). Atualização Clínica em Odontologia. Sao Paulo: Artes Medicas, 2006. cap. 14. In Meireles G C S, Santos A M. Mecanismo de ação da laserterapia sobre componentes do processo inflamatório. C\&D - Revista Eletrônica da Fainor V.3, n.1, 2010.

26. ALBERTINI, R. et. al. Effects of different protocol doses of low Power galliumaluminumarsenate (Ga-Al-As) laser radiation $(650 \mathrm{~nm})$ on carrageenan induced rat paw oedema. Journal of Photochemistry and Photobiology B: Biology, Lausanne, v. 74, n. 2-3, p. 101- 107, may 2004. In Meireles G C S, Santos A M. Mecanismo de ação da laserterapia sobre componentes do processo inflamatório. C\&D - Revista Eletrônica da Fainor V.3, n.1, 2010

27. GENOVESE, W. J. Laser de baixa intensidade: aplicações terapêuticas em Odontologia. Sao Paulo: Loise, 2000. In Meireles G C S, Santos A M. Mecanismo de ação da laserterapia sobre componentes do processo inflamatório. C\&D - Revista Eletrônica da Fainor V.3, n.1, 2010

28. Vieira WHB. Efeitos do laser de baixa intensidade sobre a performance muscular aeróbia de ratos em treinamento físico em esteira. 2004. Dissertação (mestrado em fisioterapia): Universidade Federal de São Carlos, UFSCar, São Carlos, 2004.

29. Lins RDAU, Dantas EM, Lucena KCR, Catão MHCV, Garcia AFG, Neto LGC. Efeitos bioestimulantes do laser de baixa potência no processo de reparo. An Bras Dermatol. 2010;85(6):849-55. http://dx.doi.org/10.1590/S0365-05962010000600011

30. STAINKI, D.R.; RAISER, A.G.; GRAÇA, D.L.; BECKER, C.; FERNANDEZ, G.M.S. The galium arsenide (GaAs) laser radiation in the radial nerve regeneration submitted to secundary surgical repair. Braz j vet res anim sci 1998; 35(1):37-40. 